\title{
CXCL7 promotes proliferation and invasion of cholangiocarcinoma cells
}

\author{
QIAN GUO $^{1-3}$, ZHIXIANG JIAN ${ }^{1,2}$, BAOQING JIA ${ }^{3}$ and LIANG CHANG ${ }^{4}$ \\ ${ }^{1}$ Graduate College, Southern Medical University, Guangzhou, Guangdong 510515; \\ ${ }^{2}$ Department of General Surgery, Guangdong General Hospital, Guangdong Academy of Medical Sciences, \\ Guangzhou, Guangdong 510000; ${ }^{3}$ Department of Breast Neoplasms Surgery, People's Hospital of \\ Inner Mongolia, Hohhot, Inner Mongolia 010017; ${ }^{4}$ Department of Oncology Surgery, \\ Inner Mongolia Baogang Hospital, Baotou, Inner Mongolia 404010, P.R. China
}

Received June 5, 2016; Accepted November 8, 2016

DOI: $10.3892 / o r .2016 .5312$

\begin{abstract}
CXCL7 is an important chemoattractant cytokine, which signals through binding to its receptor CXCR2. Recent studies have demonstrated that the CXCL7/CXCR2 signaling plays a promoting role in several common malignancies, including lung, renal, colon, and breast cancer. However, the regulatory role of CXCL7, in cholangiocarcinoma, as well as the underlying mechanism, has not been previously reported. Herein, we found more positive expression of CXCL7 in cholangiocarcinoma tissues compared to adjacent non-tumor tissues. High CXCL7 expression was significantly correlated with poor differentiation, lymph node metastasis, vascular invasion and advanced clinical stage, but was not associated with age, gender, or tumor size. Besides, the expression of CXCL7 was significantly associated with the Ki67 expression, but not associated with CA199, AFP, or P53 expression in cholangiocarcinoma. Moreover, the overall survival of cholangiocarcinoma patients with high CXCL7 expression was significantly shorter than those with low CXCL7 expression. In vitro study indicated that CXCL7 and CXCR2 were also positively expressed in several common cholangiocarcinoma cell lines, including HuCCT1, HuH28, QBC939, EGI-1, OZ and WITT. SiRNA-induced inhibition of CXCL7 significantly reduced the proliferation and invasion of QBC939 cells. On the contrary, overexpression of CXCL7 markedly promoted these malignant phenotypes of QBC939 cells. Of note, the conditioned medium of CXCL7-overexpresing human hepatic stellate cells could also promote the proliferation and invasion of QBC939 cells, suggesting that CXCL7 may also play an oncogenic role in cholangiocarcinoma in a paracrine-dependent manner, not
\end{abstract}

Correspondence to: Professor Zhixiang Jian, Department of General Surgery, Guangdong General Hospital, Guangdong Academy of Medical Sciences, 106 Zhongshan Er Road, Guangzhou, Guangdong 510000, P.R. China

E-mail: doctorjzx@sina.com

Key words: cholangiocarcinoma, CXCL7, proliferation, invasion only in an autocrine-dependent manner. Molecular assay data suggested that the AKT signaling pathway was involved in the CXCL7-mediated malignant phenotypes of QBC939 cells. In summary, our study suggests that CXCL7 plays a promoting role in regulating the growth and metastasis of cholangiocarcinoma.

\section{Introduction}

Cholangiocarcinoma arises from the epithelial cells of the intrahepatic, perihilar and distal biliary tree (1). Despite significant improvements in surgery combined with chemotherapy using gemcitabine and cisplatin, the prognosis of cholangiocarcinoma patients still remains poor with a median survival of less than one year (1). In the recent decade, deregulations of various oncogenes or tumor suppressors have been implicated in malignant progression of human cancers (2-4). Therefore, revealing the underlying mechanism of cholangiocarcinoma development and progression may help improve the development of effective therapeutic strategies.

Chemokines, secreted by various cell types, play central roles in chemotaxis $(5,6)$. According to the order of conserved cysteine residues, chemokines are classified into $\mathrm{C}, \mathrm{CC}, \mathrm{CXC}$ and $\mathrm{C}(\mathrm{X}) 3 \mathrm{C}$, and $\mathrm{CXC}$ chemokines are further classified into $\mathrm{ELR}^{+} \mathrm{CXC}$ and ELR-CXC, according to the absence or presence of the amino-terminal ELR motif $(6,7)$. Many studies have shown that chemokines are involved in the tumorigenesis and malignant progression of human cancers, and therefore may become potential therapeutic target for cancer treatment (8-10). For instance, lung cancer cells were found to utilize the CXCL12/CXCR4 signaling to benefit growth and distant spread (4). CXCL5/CXCR2 axis can promote the migration and invasion of bladder cancer cells by activating PI3K/AKT-induced upregulation of MMP2/MMP9 (11).

CXCL7 is a platelet-derived growth factor that belongs to the CXC chemokine family, functioning as a potent chemoattractant and activator of neutrophils through binding to its receptor CXCR2 (12). CXCL7 has been demonstrated to participate in a variety of cellular processes, such as DNA synthesis, glycolysis, mitosis, intracellular cAMP accumulation, prostaglandin E2 secretion, as well as the synthesis of 
hyaluronic acid and plasminogen activator (12-17). Moreover, it is also an antimicrobial protein with bactericidal and antifungal activity (18). Recently, CXCL7 has been found to be deregulated in human cancers, and plays a role in tumor growth. For instance, CXCL7 was found to promote the growth of clear cell renal cell carcinoma (19). Desurmont et al reported that overexpression of CXCL7 and CXCR2 in liver metastases from colon cancer was correlated to shorter disease-free and overall survival (20). However, the role of CXCL7 in cholangiocarcinoma has not been previously reported.

The present study aimed to investigate the expression of CXCL7 in cholangiocarcinoma tissues, as well as the regulatory role of CXCL7 in the malignant phenotypes of cholangiocarcinoma cells.

\section{Materials and methods}

Tissue sample collection. This study was approved by the legislation and ethical boards of Guangdong General Hospital, Guangdong Academy of Medical Sciences, Guangzhou, China. A total of 156 cholangiocarcinoma tissues and 35 adjacent non-tumor tissues were collected from our hospital. Written informed consent was obtained from all the patients. The patients involved in this study received no preoperative chemotherapy or radiotherapy. The clinicopathologic characteristics of cholangiocarcinoma samples were summarized in Table I. All tissues were formalin fixed and paraffin-embedded.

Immunohistochemical staining assay. Sections (4 $\mu \mathrm{m})$ were deparaffinized and subjected to heat-induced antigen retrieval using citrate buffer for 22 min using a microwave oven, which were then incubated with primary antibodies, and then with secondary antibody for $1 \mathrm{~h}$ at room temperature. The reaction was developed using substrate diaminobenzidine and counterstained with hematoxylin. The protein expression was scored by 3 pathologists independently. The percentage of positively staining cells was graded as 0 (no staining, negative), $+:>0$ and $\leq 25 \%$ of cells positive, $++:>25$ and $\leq 75 \%$ of cells positive, $+++:>75 \%$ of cells positive.

Cell cultures. Human cholangiocarcinoma cell lines (HuCCT1, HuH28, QBC939, EGI-1, OZ and WITT) and human hepatic stellate cell line LX-1 were purchased from Cell Bank of Chinese Academic Institute, Shanghai, China. All cell lines were cultured in DMEM (Gibco, Carlsbad, CA, USA) added with $10 \% \mathrm{FBS}$ (Gibco) in a $37^{\circ} \mathrm{C}$ incubator with $5 \% \mathrm{CO}_{2}$.

Cell transfection. Lipofectamine 2000 was used to conduct cell transfection, according to the manufacturer's instructions. Briefly, cells were cultured to $70 \%$ confluence, and resuspended in serum-free DMEM medium. The blank pcDNA3.1 vector, pcDNA3.1-CXCL7 plasmid, non-specific siRNA, CXCL7 siRNA, and Lipofectamine 2000 were diluted with serum-free medium, respectively. The diluted Lipofectamine 2000 was then added into the diluted plasmid or siRNA, and incubated for $20 \mathrm{~min}$ at room temperature, and then added into the cell suspension. After incubation at $37^{\circ} \mathrm{C}$ for $6 \mathrm{~h}$, the medium was replaced by the normal serum-containing medium. Then, cells were cultured for $48 \mathrm{~h}$ before the following assays.
Table I. Association between CXCL7 expression and clinicopathological characteristics in cholangiocarcinoma.

\begin{tabular}{|c|c|c|c|c|}
\hline Variables & No. & $\begin{array}{c}\text { Low } \\
\text { CXCL7 } \\
(n=79)\end{array}$ & $\begin{array}{c}\text { High } \\
\text { CXCL7 } \\
(n=77)\end{array}$ & P-value \\
\hline \multicolumn{5}{|l|}{ Age } \\
\hline$<55$ & 54 & 25 & 29 & 0.43 \\
\hline$\geq 55$ & 102 & 54 & 48 & \\
\hline \multicolumn{5}{|l|}{ Gender } \\
\hline Male & 94 & 50 & 44 & 0.433 \\
\hline Female & 62 & 29 & 33 & \\
\hline \multicolumn{5}{|l|}{ Tumor size } \\
\hline$<4 \mathrm{~cm}$ & 105 & 66 & 49 & 0.143 \\
\hline$\geq 4 \mathrm{~cm}$ & 51 & 23 & 28 & \\
\hline \multicolumn{5}{|l|}{ Differentiation } \\
\hline Well-Moderate & 88 & 51 & 37 & 0.038 \\
\hline Poor & 68 & 28 & 40 & \\
\hline \multicolumn{5}{|c|}{ Lymph node metastasis } \\
\hline No & 68 & 46 & 22 & 0.0002 \\
\hline Yes & 88 & 33 & 55 & \\
\hline \multicolumn{5}{|l|}{ Vascular invasion } \\
\hline No & 81 & 58 & 23 & $<0.0001$ \\
\hline Yes & 75 & 21 & 54 & \\
\hline \multicolumn{5}{|l|}{ Clinical stage } \\
\hline I-II & 61 & 44 & 17 & $<0.0001$ \\
\hline III-IV & 95 & 35 & 60 & \\
\hline
\end{tabular}

RT-PCR analysis. Total RNA was extracted by using TRIzol Reagent (Life Technologies), according to the manufacturer's instructions. A total of 800 ng RNA was converted into cDNA using Reverse Transcription kit (Life Technologies), according to the manufacturer's instructions. Real-time PCR was then performed by using Q-PCR Detection kit (Life Technologies) on ABI 7500 thermocycler. The PCR steps were $95^{\circ} \mathrm{C}$ for $10 \mathrm{~min}$, and 40 cycles of denaturation at $95^{\circ} \mathrm{C}$ for $15 \mathrm{sec}$ and annealing/elongation step at $60^{\circ} \mathrm{C}$ for $60 \mathrm{sec}$. GAPDH was used as an internal control. The relative expression was analyzed by the $2^{-\Delta \Delta C t}$ method.

Western blotting. Cells were lysed with ice-cold lysis buffer (50 mM Tris-HCl, pH 6.8, 100 mM 2-ME, 2\% w/v SDS, 10\% glycerol). Protein was separated with $10 \%$ SDS-PAGE and then transferred onto a polyvinylidene difluoride (PVDF) membrane (Life Technologies). The PVDF membrane was incubated with PBS containing $5 \%$ milk overnight at $4^{\circ} \mathrm{C}$. After washing with PBS 3 times, the PVDF membrane was incubated with primary antibodies (Abcam, Cambridge, MA, USA) at room temperature for $3 \mathrm{~h}$. After washing with PBS 3 times, the PVDF membrane was incubated with secondary antibody (Abcam) at room temperature for $1 \mathrm{~h}$. Super Signal West Pico Chemiluminescent Substrate kit (Pierce, Rockford, IL, USA) was then used to detect signals, according to the 
A

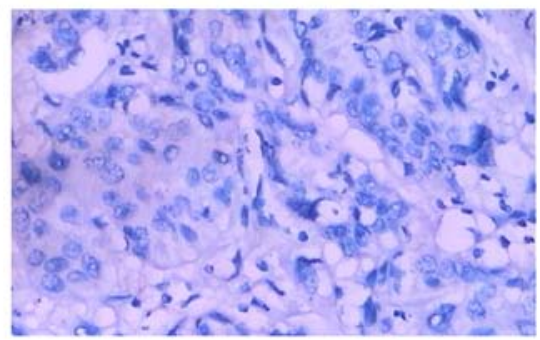

C

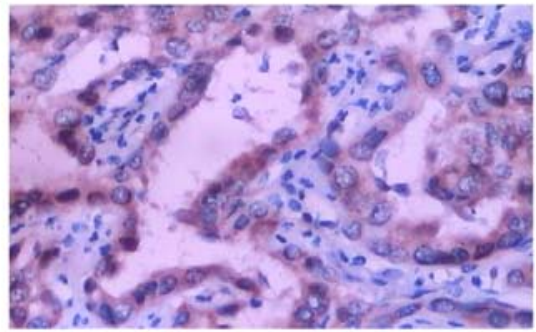

B

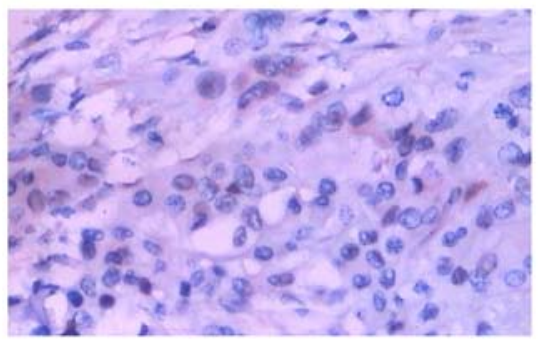

D

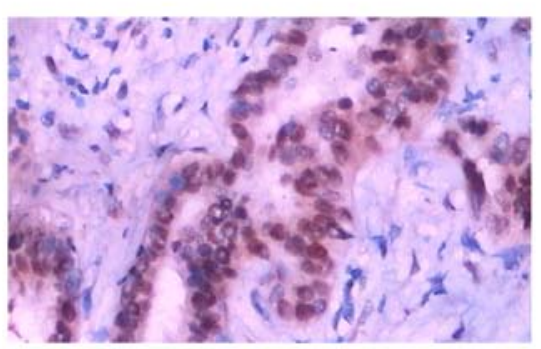

Figure 1. (A) Representative image of negative expression of CXCL7 (-). (B) Representative image of weak expression of CXCL7 (+). (C) Representative image of moderate expression of CXCL7 (++). (D) Representative image of strong expression of CXCL7 (+++). Magnification of x200.

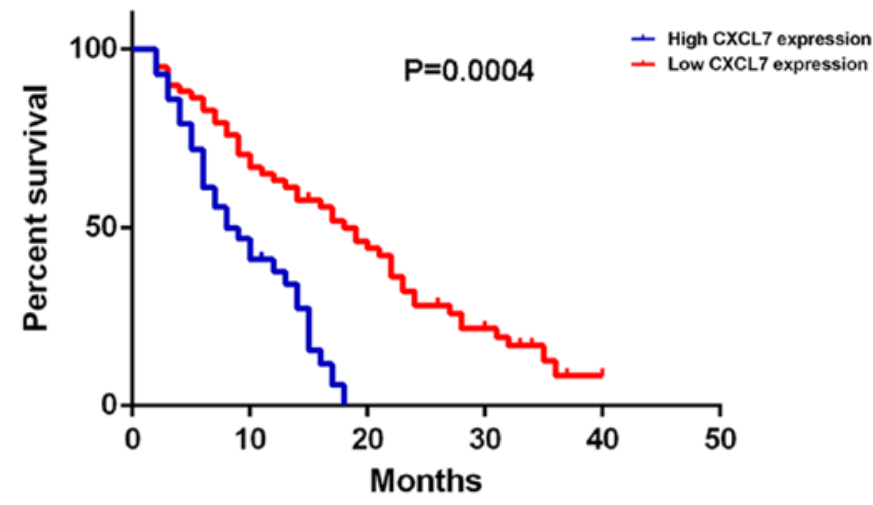

Figure 2. Cholangiocarcinoma patients with high CXCL7 expression had shorter overall survival time, when compared with those with low CXCL7 expression

manufacturer's instructions. The relative protein expression was analyzed by Image-Pro plus software 6.0, represented as the density ratio versus GAPDH.

Enzyme-linked immunosorbent assay (ELISA). Cells in DMEM containing $10 \%$ FBS were seeded in a 6-well plate $\left(1.5 \times 10^{5}\right.$ cells/well $)$ and cultured for $48 \mathrm{~h}$. Then, the supernatant was collected, and centrifuged at $12000 \mathrm{x} \mathrm{g}$ for $10 \mathrm{~min}$. The secretion level of CXCL7 was detected using a human CXCL7 ELISA kit (Thermo Fisher Scientific, Inc., Bethesda, MA, USA), according to the manufacturer's instructions.

MTT assay. MTT assay was used to examine cell proliferation. Briefly, cells were plated at a density of 10,000 cells per well in 96-well plates. After cultured for 0, 24, 48 and $72 \mathrm{~h}$, the cells were incubated with MTT at a final concentration of $0.5 \mathrm{mg} / \mathrm{ml}$ for $4 \mathrm{~h}$ at $37^{\circ} \mathrm{C}$. After the removal of the medium, $150 \mathrm{mM}$ of DMSO solution was added. The absorbance was read at $570 \mathrm{~nm}$ using a Bio-Tek ${ }^{\mathrm{TM}}$ ELX-800 ${ }^{\mathrm{TM}}$ Absorbance Microplate reader.
Transwell assay. Transwell assay was performed to examine the cell invasion using Transwell chambers (BD Biosciences, Franklin Lakes, NJ, USA). Cell suspension containing $5 \times 10^{5}$ cells $/ \mathrm{ml}$ was prepared in serum-free media, and $300 \mu \mathrm{l}$ of cell suspension was added into the upper chamber. Then, $500 \mu \mathrm{l}$ of DMEM with $10 \%$ FBS was added into the lower chamber. Cells were incubated for $24 \mathrm{~h}$. Then, a cotton-tipped swab was used to carefully wipe out the cells that did not migrate through the pores. The filters were fixed in $90 \%$ alcohol and stained by crystal violet, and observed under an inverted microscope (Olympus, Tokyo, Japan).

Statistical analysis. Data are expressed as the mean \pm SD. Statistical analysis was performed using SPSS 17.0 (SPSS, Armonk, NY, USA). The differences between two groups were analyzed using Student's t-test. The differences among more than two groups were analyzed using ANOVA. $\mathrm{P}<0.05$ indicated significant differences.

\section{Results}

Upregulation of CXCL7 is associated with cholangiocarcinoma progression. In the present study, our data indicated that the CXCL7 protein was mainly in the cytoplasm (Fig. 1). Fig. 1A represents negative expression (-), Fig. 1B weak expression (+), Fig.1C moderate expression (++), and Fig. 1D strong expression $(+++)$, respectively. The positive expression of CXCL7 was found in $66 \%(103 / 156)$ of cholangiocarcinoma cases, while only $23 \%(8 / 35)$ was detected in adjacent non-tumor tissues. We further investigated the clinical significance of CXCL7 expression in cholangiocarcinoma. The cholangiocarcinoma patients were divided into two groups, low CXCL7 expression group (negative and weak expression) and high CXCL7 expression group (moderate and strong expression). As indicated in Table I, high CXCL7 expression was significantly associated with poor differentiation, lymph node metastasis, vascular invasion, and advanced clinical stage of cholangiocarcinoma. 
A
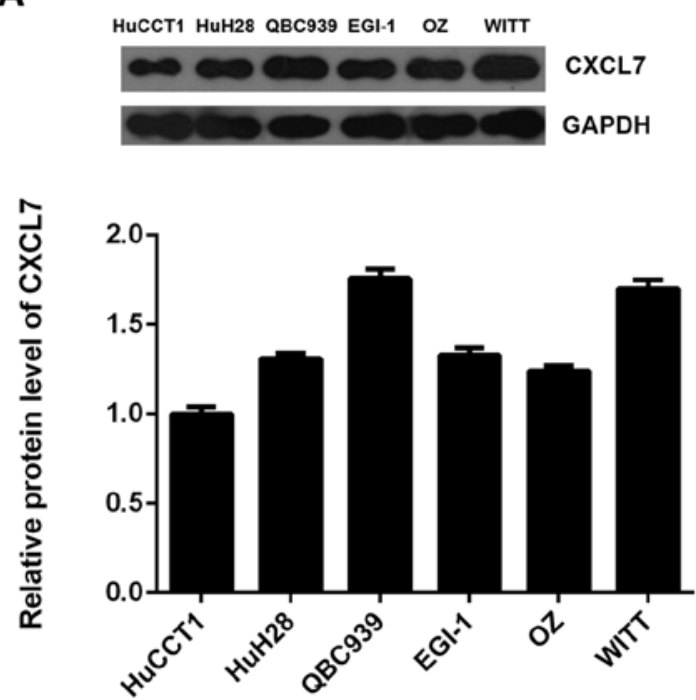

B
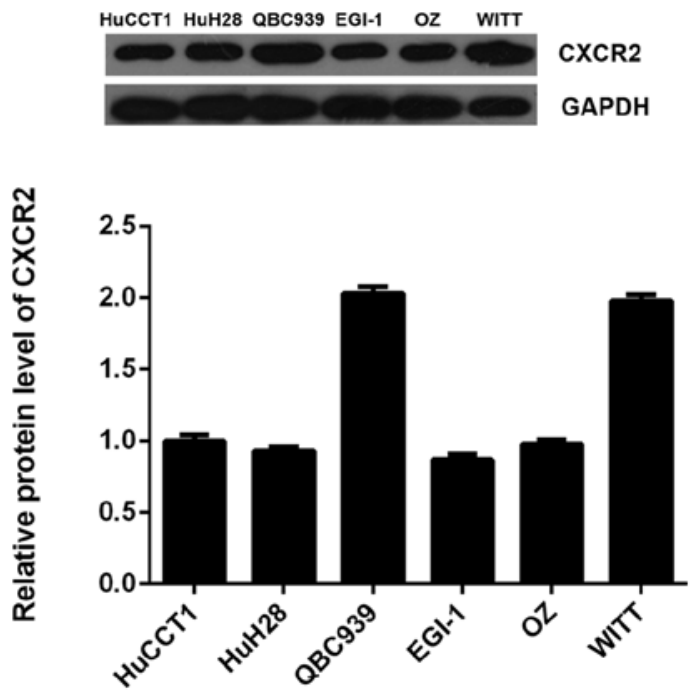

Figure 3. Western blotting was used to examine the relative protein expression of (A) CXCL7 and (B) CXCR2 in cholangiocarcinoma cell lines including HuCCT1, HuH28, QBC939, EGI-1, OZ and WITT. GAPDH was used as the internal reference.

Table II. Association between the expression levels of CXCL7 and other markers in cholangiocarcinoma.

\begin{tabular}{lrccc}
\hline Variables & No. & $\begin{array}{c}\text { Low } \\
\text { CXCL7 } \\
(\mathrm{n}=79)\end{array}$ & $\begin{array}{c}\text { High } \\
\text { CXCL7 } \\
(\mathrm{n}=77)\end{array}$ & P-value \\
\hline Ki67 & & & & \\
Negative & 50 & 38 & 12 & $<0.0001$ \\
Positive & 106 & 41 & 65 & \\
CA199 & & & & \\
Negative & 58 & 34 & 24 & 0.125 \\
Positive & 98 & 45 & 53 & \\
AFP & & & & \\
Negative & 52 & 24 & 28 & 0.428 \\
Positive & 104 & 55 & 49 & \\
P53 & & & & \\
Negative & 69 & 32 & 37 & \\
Positive & 87 & 47 & 40 & \\
\hline
\end{tabular}

However, the expression of CXCL7 was not associated with age, gender, and tumor size (Table I). We further investigated the relationship between CXCL7 protein expression and the expression of Ki67, CA199, AFP, and P53 in cholangiocarcinoma. As indicated in Table II, the expression of CXCL7 was significantly associated with the Ki67 expression. However, the CXCL7 expression was not associated with CA199, AFP, or P53 expression in cholangiocarcinoma.

Increased CXCL7 expression is associated with poor prognosis of cholangiocarcinoma patients. Further investigation indicated that the cholangiocarcinoma patients with high CXCL7 expression had shorter overall survival time, when compared with those with low CXCL7 expression (Fig. 2).
Therefore, the increased expression of CXCL7 is associated with the advanced progression and poor prognosis of patients with cholangiocarcinoma.

Knockdown of CXCL7 reduces the proliferation and invasion of cholangiocarcinoma cells. We further examined the protein expression of CXCL7 and CXCR2 in several common cholangiocarcinoma cell lines including HuCCT1, HuH28, QBC939, EGI-1, OZ and WITT. As indicated in Fig. 3A and B, the protein expression of CXCL7 and CXCR2 was positively expressed in these cholangiocarcinoma cell lines. As QBC939 cells showed the highest expression of CXCL7, we used this cell line in the following experiments.

The effects of CXCL7 on the proliferation and invasion of QBC939 cells were further studied. To knock down the expression of CXCL7, QBC939 cells were transfected with CXCL7-specific siRNA, or non-specific siRNA (NC siRNA), respectively. Our data showed that transfection with CXCL7specific siRNA significantly decreased the mRNA and protein expression of CXCL7 compared to the control group (Fig. 4A and B). MTT assay and Transwell assay further showed that knockdown of CXCL7 caused a significant decrease in the proliferation and invasion of QBC939 cells (Fig. 4C and D). These data suggest that CXCL7 plays a promoting role in the regulation of the malignant phenotypes of QBC939 cells. To further confirm these findings, QBC939 cells were transfected with pcDNA3.1-CXCL7 ORF plasmid, or blank vector as NC group, respectively. After transfection with pcDNA3.1-CXCL7 ORF plasmid, the mRNA and protein levels of CXCL7 were significantly increased, when compared to the control group, respectively (Fig. 5A and B). Moreover, overexpression of CXCL7 remarkably enhanced the proliferation and invasion of QBC939 cells (Fig. 5C and D). Taken together, we demonstrate that CXCL7 can promote the proliferation and invasion of cholangiocarcinoma cells.

CXCL7 promotes the malignant phenotypes of cholangiocarcinoma cells in a paracrine-dependent manner. As 
A

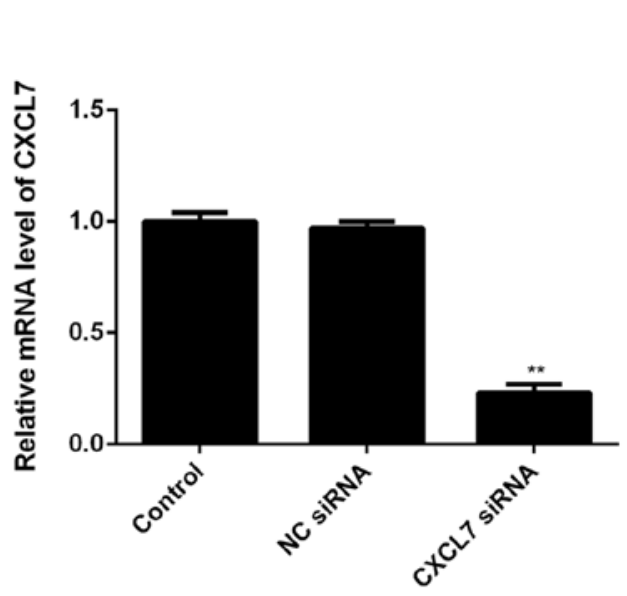

B

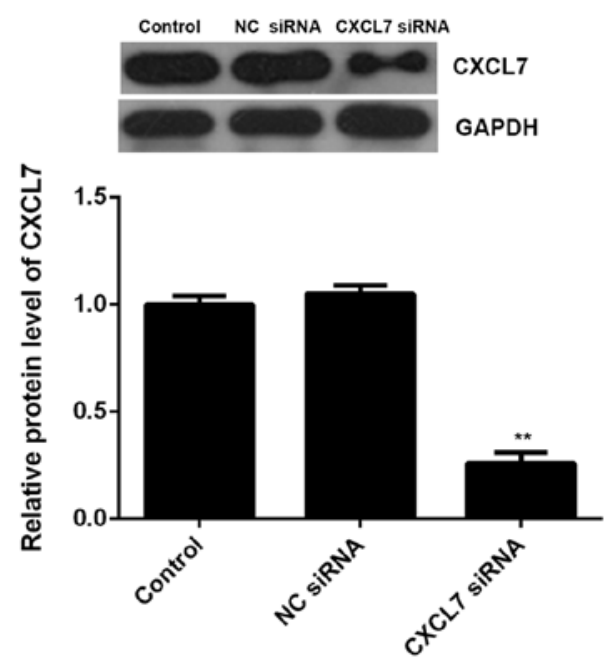

C

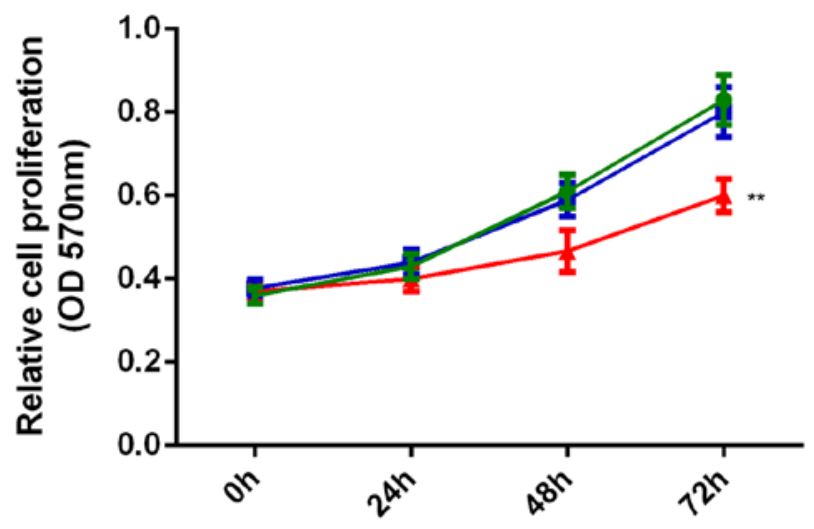

$\rightarrow$ Control

$\rightarrow$ NC siRnA

- CXCL7 SIRNA

D
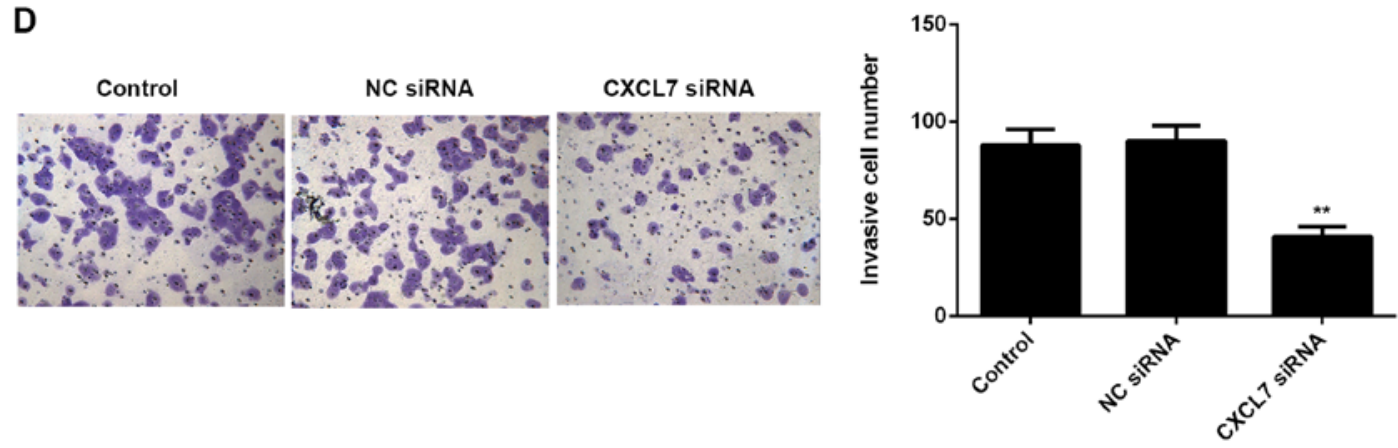

Figure 4. QBC939 cells were transfected with CXCL7-specific siRNA, or non-specific siRNA (NC siRNA), respectively. Non-transfected QBC939 cells were used as control. (A) Real-time PCR and (B) western blotting were used to examine the mRNA and protein expression of CXCL7. (C) MTT and (D) Transwell assays were used to examine the cell proliferation and invasion. ${ }^{* *} \mathrm{P}<0.01 \mathrm{vs}$. Control.

non-tumor cells in the tumor microenvironment can also secret CXCL7, we used $50 \mathrm{ng} / \mathrm{ml}$ of recombinant human CXCL7 to treat QBC939 cells for $24 \mathrm{~h}$. After treatment, the cell proliferation and invasion of QBC939 cells were examined. As shown in Fig. 6A and B, treatment with CXCL7 significantly increased the proliferation and invasion of QBC939 cells, when compared to the control group, respectively.

We further studied the effect of normal cell-derived CXCL7 on the malignant phenotypes of cholangiocarcinoma cells. Human hepatic stellate cell line LX-1 was transfected with CXCL7 ORF plasmid or blank vector, respectively. After transfection with CXCL7 ORF plasmid, the mRNA and protein expression of CXCL7 in LX-1 cells were significantly increased compared to the control group (Fig. 7A and B).
ELISA data further indicated that the CXCL7 levels in the CM of CXCL7-overexpressing LX-1 cells were higher than those in the control group (Fig. 7C). The CM of LX-1 cells were further used to culture QBC939 cells. As shown in Fig. 7D and E, the proliferation and invasion of QBC939 cells cultured with the CM of CXCL7-overexpressing LX-1 cells were significantly increased, when compared to control group, respectively. These findings confirmed that CXCL7 may also play a promoting role in cholangiocarcinoma in a paracrinedependent manner.

The AKT signaling is activated by CXCL7 in cholangiocarcinoma QBC939 cells. AKT signaling has been demonstrated to be involved in the development and malignant progression 
A

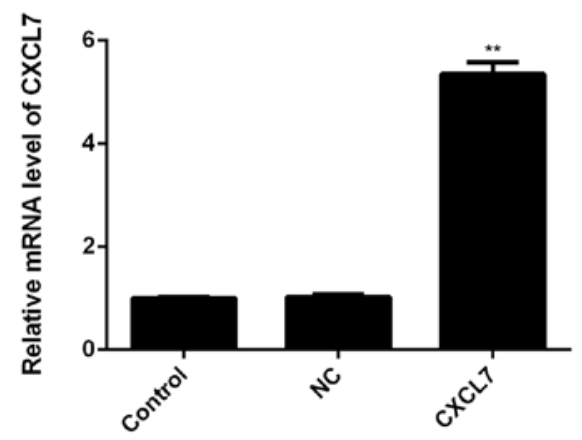

C

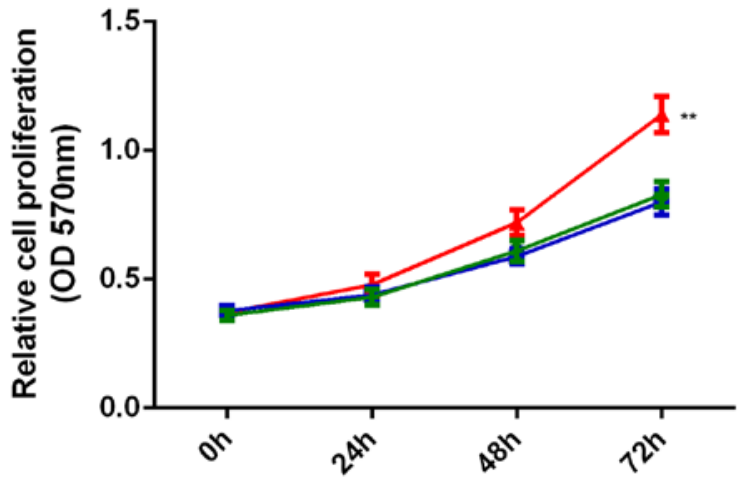

B
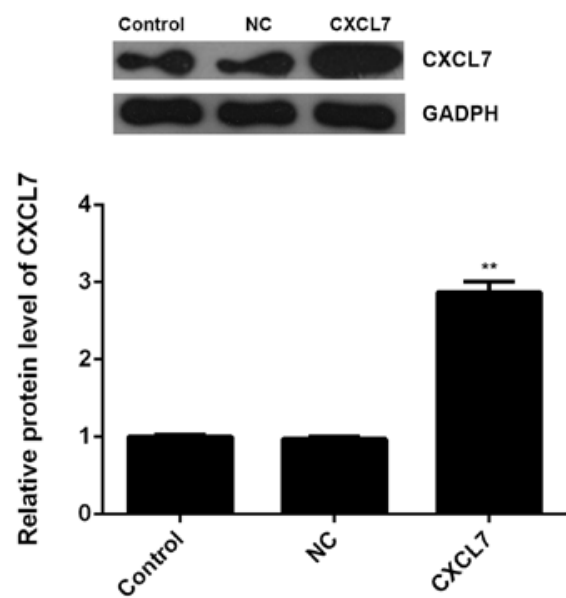

D

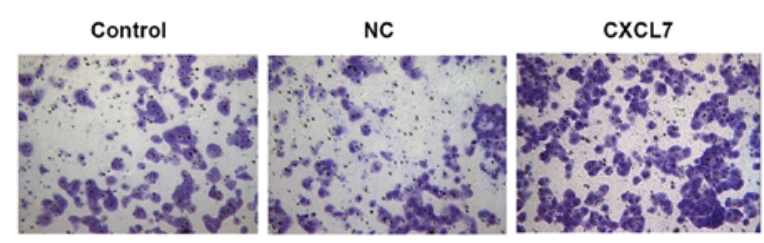

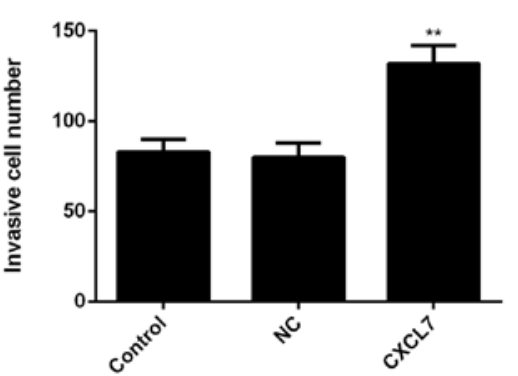

Figure 5. QBC939 cells were transfected with pcDNA3.1-CXCL7 expression plasmid, or blank vector as negative control (NC), respectively. Non-transfected QBC939 cells were used as control. (A) Real-time PCR and (B) western blotting were used to examine the mRNA and protein expression of CXCL7. (C) MTT and (D) Transwell assays were used to examine the cell proliferation and invasion. ${ }^{* * *} \mathrm{P}<0.01$ vs. Control.

of human cancers. In the present study, we studied whether the activity of AKT signaling was affected by CXCL7 in cholangiocarcinoma cells. Western blotting data showed that knockdown of CXCL7 significantly decreased the activity of AKT signaling, when compared to the control group (Fig. 8A). On the contrary, overexpression of CXCL7 enhanced its activity, when compared to the control group in cholangiocarcinoma QBC939 cells (Fig. 8B). According to these data, we suggest that the activation of AKT signaling is involved in the CXCL7-induced proliferation and invasion of cholangiocarcinoma cells.

\section{Discussion}

The clinical outcomes of cholangiocarcinoma patients remain poor. Therefore, understanding the molecular mechanism underlying cholangiocarcinoma development and progression is urgently needed, which may promote the potential benefit of targeted therapy. In the present study, we investigated the expression of CXCL7 in cholangiocarcinoma, as well as the role and molecular mechanism of CXCL7 in the regulation of the malignant phenotypes of cholangiocarcinoma cells. We found that the increased expression of CXCL7 was significantly associated with advanced progression and poor prognosis of cholangiocarcinoma patients. In vitro study showed that CXCL7 plays a promoting role in cholangiocarcinoma QBC939 cell proliferation and invasion. Moreover, treatment with both exogenous CXCL7 and the CM of CXCL7-overexpressing LX-1 cells could also promote the malignant phenotypes of QBC939 cells. In addition, the activity of AKT signaling was found to be upregulated after CXCL7 overexpression.

Deregulations of CXCL7 have been observed in several human cancers, and it generally plays an oncogenic role. For instance, CXCL7 is an independent prognostic factor for overall survival in clear cell renal cell carcinoma (ccRCC). Moreover, it was also reported that SB225002, an inhibitor of CXCR1 and CXCR2, could inhibit endothelial cell prolifera- 
A
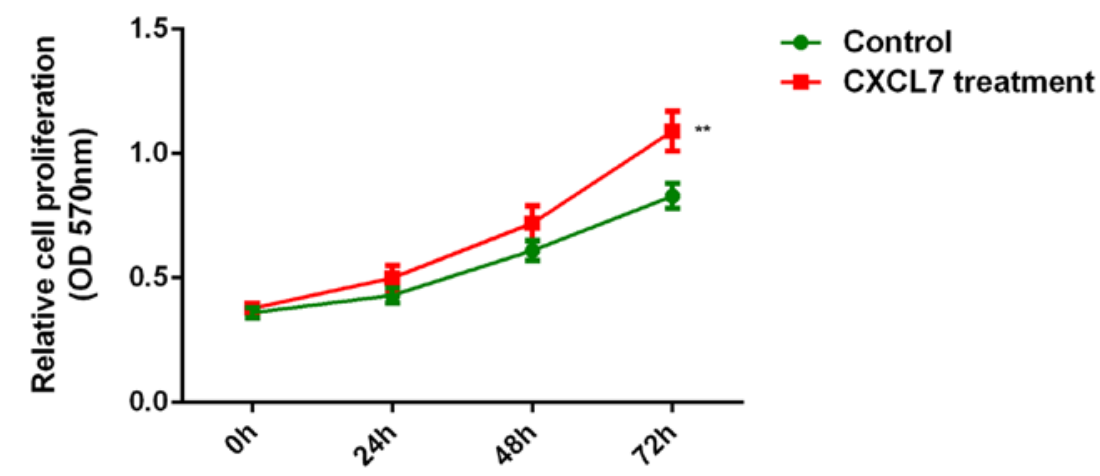

B
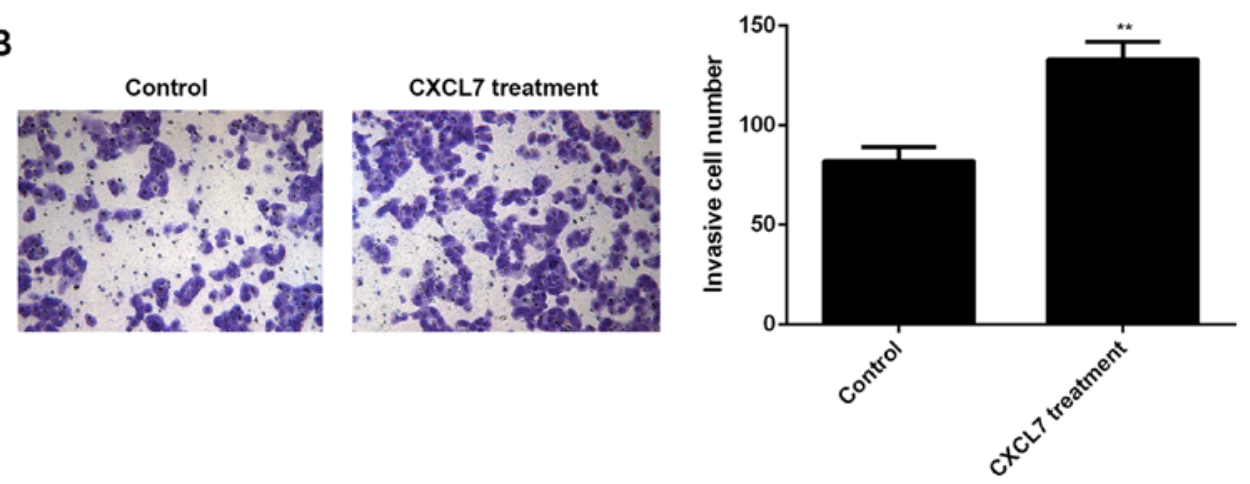

Figure 6. QBC939 cells were treated with $50 \mathrm{ng} / \mathrm{ml}$ of recombinant human CXCL7 for $24 \mathrm{~h}$. Non-treated cells were used as control. (A) MTT and (B) Transwell assays were used to examine the cell proliferation and invasion. ${ }^{* *} \mathrm{P}<0.01$ vs. Control.

tion, tumor angiogenesis and ccRCC growth. On the contrary, overexpression of CXCL7 enhanced ccRCC cell proliferation in vitro and tumor growth in vivo (19). Here we for the first time reported that CXCL7 was mainly expressed in cholangiocarcinoma tissues compared to adjacent non-tumor tissues, and the increased expression of CXCL7 was significantly associated with poor differentiation, vascular invasion, lymph node metastasis, advanced clinical stage, as well as shorter overall survival time, but was not associated with age, gender and tumor size. We further showed that CXCL7 and its receptor CXCR2 were positively expressed in several common cholangiocarcinoma cell lines. Therefore, CXCL7 may play a promoting role in the malignant progression of cholangiocarcinoma.

We further studied the regulatory role of CXCL7 in cholangiocarcinoma cells. Cholangiocarcinoma QBC939 cells were transfected with CXCL7-specific siRNA or ORF plasmid to downregulate or upregulate its expression, respectively. We found that knockdown of CXCL7 significantly decreased QBC939 cell proliferation and invasion. On the contrary, overexpression of CXCL7 remarkably promoted these cellular events of QBC939 cells. These findings suggest that CXCL7 may play a promoting role in cholangiocarcinoma growth and metastasis. Similar findings were also reported in other cancer types. For instance, tumors established from Lewis lung carcinoma (LLC) cells overexpressing CXCL7 increased the infiltration of M2 macrophages at the early stages of tumorigenesis, and these CXCL7-overexpressing LLC tumors developed faster than control tumors, suggesting that CXCL7 attracts macrophages especially at the tumor site and may accelerate lung tumor development in the early stages (21).
Therefore, CXCL7 may become a potential therapeutic target for human cancers including cholangiocarcinoma.

As CXCL7 could also be secreted by other normal cell types in tumor microenvironment, we studied whether CXCL7 could also promote the malignant phenotypes of cholangiocarcinoma cells in a paracrine-dependent manner. Recombinant human CXCL7 was used to treat QBC939 cells, and the exogenous CXCL7 was able to promote proliferation and invasion of QBC939 cells. Moreover, we used the CM of CXCL7-overexpressing LX-1 cells to culture the QBC939 cells, and found that the CM of CXCL7-overexpressing LX-1 cells significantly enhanced the malignant phenotypes of QBC939 cells. These findings indicate that CXCL7 also plays a promoting role in cholangiocarcinoma in a paracrinedependent manner.

Moreover, we studied the alteration of the AKT signaling in cholangiocarcinoma cells after CXCL7 overexpression. AKT signaling pathway is present in all cells of higher eukaryotes and is highly conserved. Activated Akt participates in the regulation of such cellular processes as cell growth, proliferation, survival, migration, and angiogenesis, by phosphorylating a range of intracellular proteins $(22,23)$. Moreover, it has been found to play a key role in tumor growth and metastasis, and thus can become an important target for cancer treatment $(24,25)$. Wilson et al reported that inhibition of the AKT signaling pathway suppressed cell viability via induction of apoptosis in cholangiocarcinoma (26). Huang et al also showed that FHIT suppresses proliferation and promotes apoptosis in cholangiocarcinoma cells by blockage of Akt signaling pathway (27). Besides, dual inhibition of AKT and ERK 


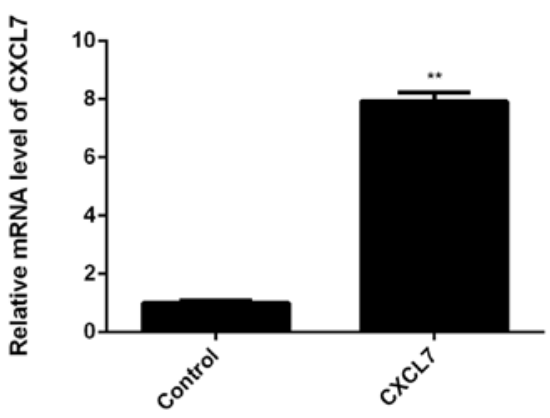

C

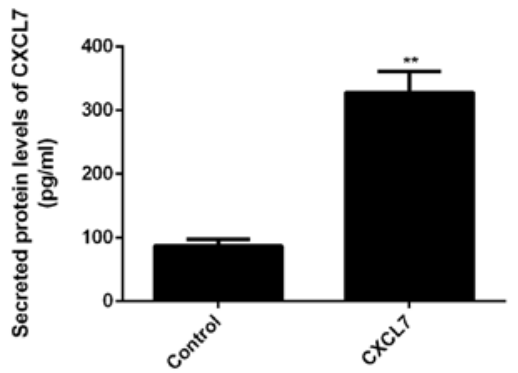

E

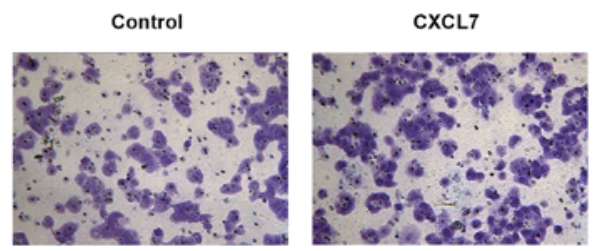

B
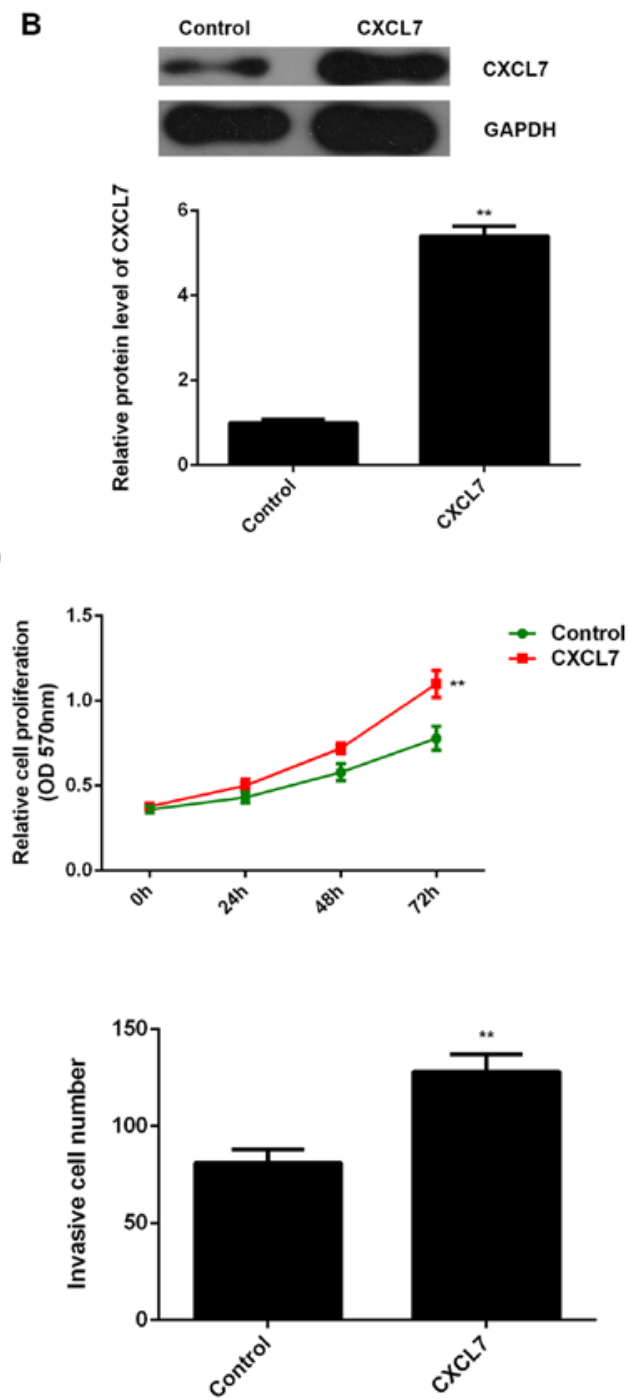

Figure 7. Human hepatic stellate cell line LX-1 was transfected with pcDNA3.1-CXCL7 expression plasmid, or blank vector as Control, respectively. (A) Real-time PCR, (B) western blotting, and (C) ELISA were used to examine the mRNA, protein, and secretion levels of CXCL7, respectively. The CM of LX-1 cells were further used to culture QBC939 cells. (D) MTT assay and (E) Transwell assay were used to examine the proliferation and invasion of QBC939 cells. ${ }^{* *} \mathrm{P}<0.01$ vs. control.

A
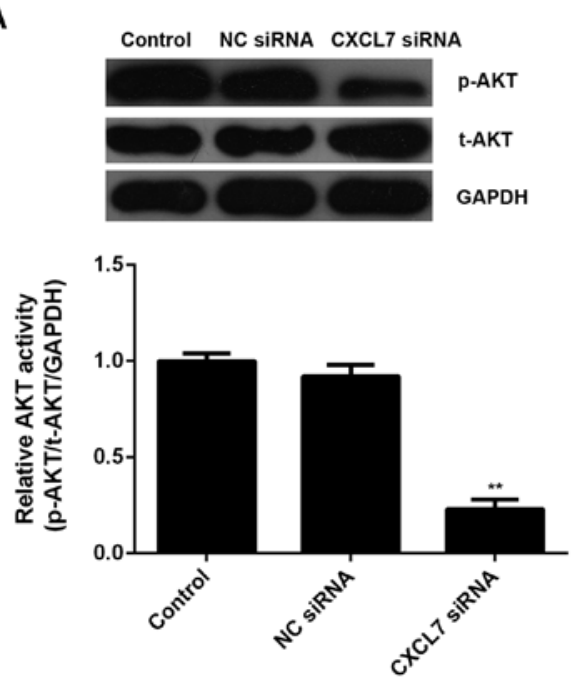

B
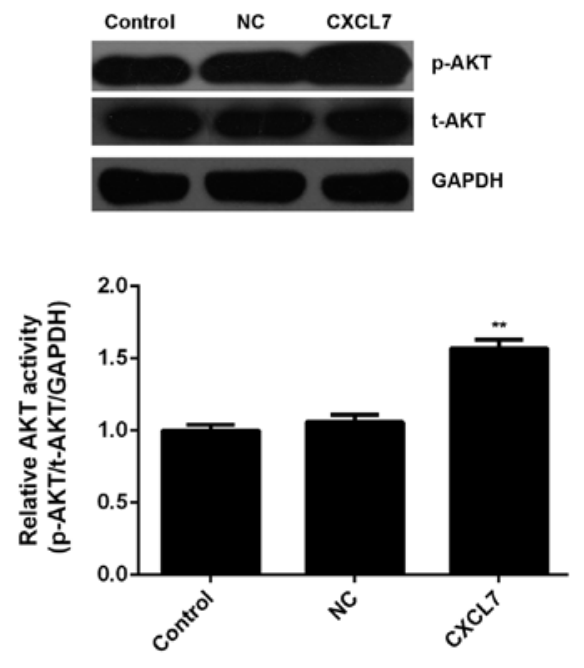

Figure 8. (A) QBC939 cells were transfected with CXCL7-specific siRNA, or non-specific siRNA (NC siRNA), respectively. (B) QBC939 cells were transfected with pcDNA3.1-CXCL7 expression plasmid, or blank vector as negative control (NC), respectively. Non-transfected QBC939 cells were used as control. Western blotting were used to examine the protein levels of phosphorylated AKT (p-AKT) and total AKT (t-AKT). GAPDH was used as the internal reference. The relative activity of AKT signaling was then determined. ${ }^{* *} \mathrm{P}<0.01$ vs. Control. 
signaling is synergistic in cholangiocarcinoma and reverses acquired resistance to MEK-inhibitors (28). In the present study, we found that overexpression of CXCL7 enhanced the activity of AKT signaling in cholangiocarcinoma cells, suggesting that this signaling pathway is probably involved in the CXCL7-mediated malignant phenotypes of cholangiocarcinoma cells.

In conclusion, the present study suggests that CXCL7 plays promoting role in the proliferation and invasion of cholangiocarcinoma cells through activation of AKT signaling pathways, and blockage of the connection between cholangiocarcinoma and adjacent tissue may be an effective strategy for the treatment of cholangiocarcinoma.

\section{References}

1. Chong DQ and Zhu AX: The landscape of targeted therapies for cholangiocarcinoma: Current status and emerging targets. Oncotarget: Apr 18, 2016 (Epub ahead of print).

2. Andersen JB: Molecular pathogenesis of intrahepatic cholangiocarcinoma. J Hepatobiliary Pancreat Sci 22: 101-113, 2015.

3. Ching CB and Hansel DE: Expanding therapeutic targets in bladder cancer: The PI3K/Akt/mTOR pathway. Lab Invest 90: 1406-1414, 2010

4. Wang Z, Sun J, Feng Y, Tian X, Wang B and Zhou Y: Oncogenic roles and drug target of CXCR4/CXCL12 axis in lung cancer and cancer stem cell. Tumour Biol 37: 8515-8528, 2016.

5. Guo N, Liu F, Yang L, Huang J, Ding X and Sun C: Chemokine receptor 7 enhances cell chemotaxis and migration of metastatic squamous cell carcinoma of head and neck through activation of matrix metalloproteinase-9. Oncol Rep 32: 794-800, 2014.

6. van der Vorst EP, Döring Y and Weber C: Chemokines. Arterioscler Thromb Vasc Biol 35: e52-e56, 2015.

7. Ding J and Tredget EE: The role of chemokines in fibrotic wound healing. Adv Wound Care (New Rochelle) 4: 673-686, 2015.

8. Duda DG, Kozin SV, Kirkpatrick ND, Xu L, Fukumura D and Jain RK: CXCL12 (SDF1alpha)-CXCR4/CXCR7 pathway inhibition: An emerging sensitizer for anticancer therapies? Clin Cancer Res 17: 2074-2080, 2011.

9. Kowalczuk O, Burzykowski T, Niklinska WE, Kozlowski M, Chyczewski L and Niklinski J: CXCL5 as a potential novel prognostic factor in early stage non-small cell lung cancer: Results of a study of expression levels of 23 genes. Tumour Biol 35: 4619-4628, 2014.

10. Rehman AO and Wang CY: CXCL12/SDF-1 alpha activates NF-kappaB and promotes oral cancer invasion through the Carma3/Bcl10/Malt1 complex. Int J Oral Sci 1: 105-118, 2009.

11. Gao Y, Guan Z, Chen J, Xie H, Yang Z, Fan J, Wang X and Li L: CXCL5/CXCR2 axis promotes bladder cancer cell migration and invasion by activating PI3K/AKT-induced upregulation of MMP2/MMP9. Int J Oncol 47: 690-700, 2015.

12. von Hundelshausen P, Petersen F and Brandt E: Platelet-derived chemokines in vascular biology. Thromb Haemost 97: 704-713, 2007.

13. Kalwitz G, Neumann K, Ringe J, Sezer O, Sittinger M, Endres M and Kaps C: Chondrogenic differentiation of human mesenchymal stem cells in micro-masses is impaired by high doses of the chemokine CXCL7. J Tissue Eng Regen Med 5: 50-59, 2011.
14. Blunk JA, Sauerstein K and Schmelz M: Experimental thermal lesions induce beta-thromboglobulin release from activated platelets. Eur J Pain 15: 23-28, 2011.

15. Zhuang P, Wo D, Xu ZG, Wei W and Mao HM: Dynamic changes in plasma tissue plasminogen activator, plasminogen activator inhibitor-1 and beta-thromboglobulin content in ischemic stroke. J Clin Neurosci 22: 1123-1127, 2015.

16. Tai PK, Liao JF, Hossler PA, Castor CW and Carter-Su C: Regulation of glucose transporters by connective tissue activating peptide-III isoforms. J Biol Chem 267: 19579-19586, 1992.

17. Kwiatkowski S, Czajka R, Dołegowska B, Chlubek D and Torbé A: Evaluation of neutrophile elastase and isoprostane 8epiPGF2alpha concentrations in maternal and umbilical cord blood serum and in amniotic fluid in pregnancies complicated by premature rupture of membranes. Ginekol Pol 79: 281-286, 2008 (In Polish).

18. González-Cortés C, Diez-Tascón C, Guerra-Laso JM, GonzálezCocaño MC and Rivero-Lezcano OM: Non-chemotactic influence of CXCL7 on human phagocytes. Modulation of antimicrobial activity against $L$. pneumophila. Immunobiology 217 : 394-401, 2012.

19. Grépin R, Guyot M, Giuliano S, Boncompagni M, Ambrosetti D, Chamorey E, Scoazec JY, Negrier S, Simonnet H and Pagès G: The CXCL7/CXCR1/2 axis is a key driver in the growth of clear cell renal cell carcinoma. Cancer Res 74: 873-883, 2014

20. Desurmont T, Skrypek N, Duhamel A, Jonckheere N, Millet G, Leteurtre E, Gosset P, Duchene B, Ramdane N, Hebbar M, et al: Overexpression of chemokine receptor CXCR2 and ligand CXCL7 in liver metastases from colon cancer is correlated to shorter disease-free and overall survival. Cancer Sci 106: 262-269, 2015.

21. Unver N, Esendagli G, Yilmaz G and Guc D: CXCL7-induced macrophage infiltration in lung tumor is independent of CXCR2 expression: CXCL7-induced macrophage chemotaxis in LLC tumors. Cytokine 75: 330-337, 2015.

22. Zhang J, Yu XH, Yan YG, Wang C and Wang WJ: PI3K/Akt signaling in osteosarcoma. Clin Chim Acta 444: 182-192, 2015.

23. Li B, Qiu T, Zhang P, Wang X, Yin Y and Li S: IKVAV regulates ERK $1 / 2$ and Akt signalling pathways in BMMSC population growth and proliferation. Cell Prolif 47: 133-145, 2014.

24. Qin Y, Cui W, Yang X and Tong B: Kaempferol inhibits the growth and metastasis of cholangiocarcinoma in vitro and in vivo. Acta Biochim Biophys Sin (Shanghai) 48: 238-245, 2016.

25. Zhou SL, Zhou ZJ, Hu ZQ, Li X, Huang XW, Wang Z, Fan J, Dai $Z$ and Zhou J: CXCR2/CXCL5 axis contributes to epithelialmesenchymal transition of HCC cells through activating PI3K/ Akt/GSK-33/Snail signaling. Cancer Lett 358: 124-135, 2015.

26. Wilson JM, Kunnimalaiyaan S, Kunnimalaiyaan M and Gamblin TC: Inhibition of the AKT pathway in cholangiocarcinoma by MK2206 reduces cellular viability via induction of apoptosis. Cancer Cell Int 15: 13, 2015.

27. Huang Q, Liu Z, Xie F, Liu C, Shao F, Zhu CL and Hu S: Fragile histidine triad (FHIT) suppresses proliferation and promotes apoptosis in cholangiocarcinoma cells by blocking PI3K-Akt pathway. Sci World J 2014: 179698, 2014.

28. Ewald F, Nörz D, Grottke A, Hofmann BT, Nashan B and Jücker M: Dual inhibition of PI3K-AKT-mTOR- and RAF-MEKERK-signaling is synergistic in cholangiocarcinoma and reverses acquired resistance to MEK-inhibitors. Invest New Drugs 32: 1144-1154, 2014 